\title{
Genomic complexity of urothelial bladder cancer revealed in urinary cfDNA
}

\author{
Fiona S Togneri ${ }^{1}$, Douglas G Ward ${ }^{2}$, Joseph M Foster ${ }^{3}$, Adam J Devall ${ }^{2}$, Paula Wojtowicz ${ }^{1}$, Sofia Alyas ${ }^{1}$,
} Fabiana Ramos Vasques ${ }^{1}$, Assa Oumie ${ }^{3}$, Nicholas D James ${ }^{4}$, KK Cheng ${ }^{5}$, Maurice P Zeegers ${ }^{6}$, Nayneeta Deshmukh ${ }^{2}$, Brendan O'Sullivan ${ }^{7}$, Philippe Taniere ${ }^{7}$, Karen G Spink ${ }^{3}$, Dominic J McMullan ${ }^{1}$, Mike Griffiths ${ }^{1}$ and Richard T Bryan ${ }^{\star 2}$

Urothelial bladder cancers (UBCs) have heterogeneous clinical characteristics that are mirrored in their diverse genomic profiles. Genomic profiling of UBCs has the potential to benefit routine clinical practice by providing prognostic utility above and beyond conventional clinicopathological factors, and allowing for prediction and surveillance of treatment responses. Urinary DNAs representative of the tumour genome provide a promising resource as a liquid biopsy for non-invasive genomic profiling of UBCs. We compared the genomic profiles of urinary cellular DNA and cell-free DNA (cfDNA) from the urine with matched diagnostic formalin-fixed paraffin-embedded tumour DNAs for 23 well-characterised UBC patients. Our data show urinary DNAs to be highly representative of patient tumours, allowing for detection of recurrent clinically actionable genomic aberrations. Furthermore, a greater aberrant load (indicative of tumour genome) was observed in cfDNA over cellular DNA $(P<0.001)$, resulting in a higher analytical sensitivity for detection of clinically actionable genomic aberrations $(P<0.04)$ when using cfDNA. Thus, cfDNA extracted from the urine of UBC patients has a higher tumour genome burden and allows greater detection of key genomic biomarkers $(90 \%)$ than cellular DNA from urine $(61 \%)$ and provides a promising resource for robust whole-genome tumour profiling of UBC with potential to influence clinical decisions without invasive patient interventions. European Journal of Human Genetics (2016) 24, 1167-1174; doi:10.1038/ejhg.2015.281; published online 13 January 2016

\section{INTRODUCTION}

Urothelial bladder cancer (UBC) is the seventh most common cancer in Western societies with a rising global incidence. ${ }^{1}$ Disease management poses numerous challenges because of the following: (i) the propensity for non-muscle-invasive bladder cancer (NMIBC) to recur, necessitating long-term surveillance; (ii) a variable risk of NMIBC progression, associated with poor 5-year survival; ${ }^{2,3}$ (iii) a lack of proven biomarker prognosticators to identify those subsets of patients who will suffer tumour recurrence, progression and death; and (iv) the radical therapies required to treat muscle-invasive disease (MIBC). ${ }^{4}$ UBCs are thus highly heterogeneous in their clinical characteristics and this is mirrored in their genomics, characteristics of which traverse conventional grade and stage groupings. ${ }^{5}$

Typically, genomic aberrations in tumours have been characterised using formalin-fixed paraffin-embedded (FFPE) or fresh-frozen tumour tissue, with such analyses elucidating promising biomarkers and suggesting genomic signatures with potential to influence future therapeutic interventions. ${ }^{6-8}$ Identifying such genomic complexity in a non-invasive manner could be highly advantageous for facilitating the diagnosis, treatment and surveillance of patients with NMIBC or MIBC. ${ }^{9,10}$

Genetic changes in UBCs have previously been investigated noninvasively using genetic material present in the urine. Both genetic material from exfoliated cells (which pellet upon centrifugation) and cell-free DNA (cfDNA; which remains in the supernatant following centrifugation) have been studied. Most studies to date have focused on exfoliated cells, with data giving a specific read out, for example, the presence or absence of UBC. ${ }^{11}$ Urine tests looking at genomic copy number $(\mathrm{CN})$ include the FISH-based UroVysion test (Abbott, Des Plaines, IL, USA; FDA-approved UBC diagnosis), ${ }^{12}$ which uses individual exfoliated tumour cells isolated from urine, and the CGH-based BCA-1 test, which uses DNA extracted from these exfoliated cells. BCA-1 has been used to examine more detailed $\mathrm{CN}$ data in bladder cancer patients than that provided by UroVysion, and shows some promise. ${ }^{13,14}$ Unfortunately, obtaining sufficient cellular material for analysis is not always possible, hindering the clinical applicability of such tests. A small number of studies have therefore also investigated urinary cfDNA for UBC analysis with mixed results, and it has previously been suggested that due to its origin, cfDNA may be enriched for tumour-specific biomarkers with reduced contamination from germline DNA of non-cancerous cells. ${ }^{15}$

cfDNA in blood plasma, arising through cancer cell death (necrotic or apoptotic cells) and actively released DNA, ${ }^{16,17}$ has been well studied as a liquid biopsy for various solid tumours. cfDNA in urine of bladder cancer patients has also been studied in this setting. ${ }^{15}$ This nucleic acid resource has been proposed to be predominantly necrotic in origin and quantitative changes in necrotic-specific

\footnotetext{
${ }^{1}$ West Midland Regional Genetics Laboratory, Birmingham Women's NHS Foundation Trust, Birmingham, UK; ${ }^{2}$ Institute of Cancer \& Genomic Sciences, College of Medical and Dental Sciences, University of Birmingham, Birmingham, UK; ${ }^{3}$ Affymetrix UK Ltd, High Wycombe, UK; ${ }^{4}$ Cancer Research Unit, University of Warwick, Coventry, UK; ${ }^{5}$ School of Health and Population Sciences, University of Birmingham, Birmingham, UK; ${ }^{6}$ Department of Complex Genetics, NUTRIM School of Nutrition and Translational Research in Metabolism, Maastricht University Medical Centre, The Netherlands; ${ }^{7}$ Department of Histopathology, University Hospitals Birmingham NHS Foundation Trust, Birmingham, UK ${ }^{*}$ Correspondence: Dr RT Bryan, Institute of Cancer \& Genomic Sciences, College of Medical and Dental Sciences, University of Birmingham, Edgbaston, Birmingham B15 2TT, UK. Tel: +44 0121414 7870; E-mail: r.t.bryan@bham.ac.uk

Received 2 June 2015; revised 26 November 2015; accepted 8 December 2015; published online 13 January 2016
} 
cfDNA levels have been studied to discriminate between cancer and non-cancer patients. ${ }^{18}$

In this study, we report the utilisation of Affymetrix's OncoScan FFPE Assay Kit (Affymetrix, Santa Clara, CA, USA) for detailed genomic profiling of UBC using matched FFPE tumour-derived DNA, cellular DNA from urine cell pellets and cfDNA from urine supernatant. We demonstrate that the complex genomics and important clinically actionable aberrations that are evident in FFPE tumour material (currently the predominant diagnostic biospecimen for solid tumours) are echoed in urinary DNAs, and that the tumour genome is enriched in cfDNA compared with cellular DNA. These data illustrate that urinary cfDNA may represent a reliable resource for non-invasive genomic profiling of bladder cancer.

\section{MATERIALS AND METHODS}

\section{Patients and biospecimens}

FFPE tissue and urine samples were selected from the Bladder Cancer Prognosis Programme biospecimen repository on the basis of availability of matched FFPE, urine cell pellets and $>5 \mathrm{ml}$ urine supernatant for each patient (BCPP, ethics approval 06/MRE04/65). ${ }^{19}$ Patients were enrolled into BCPP on the basis of initial cystoscopic findings suggestive of primary UBC. All patients were newly diagnosed, had not received treatment for UBC before biospecimen collection and were subsequently treated according to contemporary guidelines. Inclusion and exclusion criteria are detailed elsewhere. ${ }^{19}$

Urine samples were obtained before transurethral resection of bladder tumour(s) (TURBT). Samples were placed on ice, centrifuged at 2000 r.p.m. for $10 \mathrm{~min}$ within $8 \mathrm{~h}$ of collection, and the supernatants and cell pellets were separated and stored at $-80^{\circ} \mathrm{C}$. Representative FFPE samples were retrieved from local histopathology departments after clinical utilisation had ceased. Tumour grade and stage records were amended according to results of re-resection or cystectomy (where performed), and $10 \%$ of all FFPE samples collected underwent expert pathological review as part of routine quality assurance.

As patients were recruited to the $\mathrm{BCPP}$ cohort before a definitive diagnosis from TURBT, several patients were later found not to have UBC. Urinary supernatants from 12 of these patients were additionally included in this study as non-UBC controls and analysed alongside samples from the 23 confirmed UBC patients.

\section{DNA extraction}

For the extraction of DNA from FFPE, seven sections were cut from blocks and mounted on Superfrost slides (Thermo Fisher Scientific, Waltham, MA, USA). The fourth section from each block was cut with a thickness of $3 \mu \mathrm{m}$ and stained with haematoxylin and eosin to identify tumour-enriched regions. This section was used as a template for macrodissection of the tumour-enriched region from the remaining unstained 6- $\mu \mathrm{m}$ sections. DNA was extracted using the QIAamp DNA FFPE Tissue Kit (QIAGEN, Venlo, Netherlands), following the manufacturer's protocol.

Cellular DNA was extracted from urine pellets using the Urine DNA Isolation Kit for Exfoliated Cells or Bacteria (Norgen Biotek Corporation, ON, Canada) following the manufacturer's protocol. cfDNA was extracted from the supernatant of centrifuged urine using the Urine DNA Isolation Kit (Slurry Format; Norgen Biotek Corporation), following the manufacturer's protocol. Volumes available for extraction varied per patient, range of 6-27 ml (average of $18 \mathrm{ml}$ ).

DNAs from FFPE blocks, and cellular and cfDNA from urine were quantified using the Quant-iT PicoGreen dsDNA Assay Kit (Life Technologies, Carlsbad, CA, USA) following the manufacturer's protocol. The concentration of DNA stock was adjusted towards the recommended DNA input of $12 \mathrm{ng} / \mu \mathrm{l}$. Depending on the concentration of extracted DNAs, samples were diluted using reduced-EDTA TE buffer (10 mm Tris-HCl, 0.1 mm disodium EDTA, $\mathrm{pH}$ 8 ), concentrated using vacuum evaporation (where initial concentration was $<12>2 \mathrm{ng} / \mu \mathrm{l})$ or concentrated by reprecipitation with sodium acetate and ethanol (where initial concentration was $<2 \mathrm{ng} / \mu \mathrm{l}$ ).

\section{OncoScan assay}

Up to $12 \mathrm{ng} / \mu$ l DNA was plated at $6.6 \mu \mathrm{l}$ per well (maximum of $79.2 \mathrm{ng}$ DNA per well) into MicroAmp Optical 96-well reaction plates (Life Technologies), which were either used immediately or frozen at $-20^{\circ} \mathrm{C}$ until needed.

The OncoScan assay utilises molecular inversion probe (MIP) technology, ${ }^{20}$ for the identification of $\mathrm{CN}$ alterations, loss of heterozygosity (LOH) and recurrent clinically actionable somatic mutations (SMs). MIP probes in this assay enable the capture of the alleles of over 220000 SNPs distributed across the whole genome, with increased probe density within $\sim 900$ cancer genes. They also enable detection of 74 frequently tested somatic mutations in BRAF, KRAS, EGFR, IDH1, IDH2, PTEN, PIK3CA, NRAS and TP53. The assay was undertaken following the recommended OncoScan protocol as previously described. ${ }^{21}$

OSCHP files were generated by the OncoScan Console software (Affymetrix), using data from fluorescence intensity (CEL) files generated during scanning of OncoScan chips. OSCHP files were used as inputs for the SM Viewer Software v1.01.16304 (Affymetrix) for the detection of SMs in each sample and Nexus Express for OncoScan 3.0.1 (BioDiscovery, Hawthorne CA, USA) for the analysis of $\mathrm{CN}$ aberrations and $\mathrm{LOH}$.

\section{Data analysis}

CN (loss, gain, biallelic loss or high amplification) and LOH calls were made in each sample independently, using the TuScan algorithm supplied with the OncoScan Console, followed by blinded manual interpretation of complex profiles. Grouped comparative analyses were then performed per patient to ensure consistent genome alignment for matched samples (some samples were reprocessed using BioDiscovery's FASST2 algorithm and traces realigned as required). These grouped analyses additionally allowed for confirmation of related genomics and a late stage sample identity check. SM calls were made separately using the SM Viewer software before genomic data were combined to give full genomic profiles. Somatic mutations identified in each patient were validated by next-generation sequencing technologies, subject to availability of material. Repeat calling of matched aberrations in corresponding patient samples allowed for further confirmation of findings. Before this study, a multi-site detailed platform validation study was completed. Results of this validation further inform the reliability of the OncoScan's genomic data. ${ }^{21}$

Following $\mathrm{CN}$ profile processing, $\mathrm{CN}$ aberrations that were present in matched cfDNA and cellular DNA samples were identified. The integer $\mathrm{CN}$ for each of these aberrations was estimated under the assumption of a normal diploid cell population mixed with a single homogeneous tumour clone. Aberrant cell fraction was then calculated on an aberration by aberration basis by formulas specific to the integer $\mathrm{CN}$ state of the aberration. This resulted in no fewer than three aberrations and their calculated aberrant cell fractions describing each matched set. To test whether the mean aberrant cell fraction of cfDNA was greater than that of cellular DNA, a one-sided paired $t$-test was performed, with the null hypothesis that there were no differences in the mean aberrant cell fraction between cfDNA and cellular DNA.

Genomic profiles were mined for the presence of genomic biomarkers listed in Van Allen's database of 'tumour alterations relevant for genomics driven therapy' (TARGET: alterations that may have therapeutic, prognostic or diagnostic implications). ${ }^{22}$ Using the FFPE genomic profiles as the reference, the analytical sensitivities (for detection of FFPE-identified TARGET aberrations) were determined for each of the cfDNA and cellular DNA. The percentage sensitivities for each urinary DNA component were then compared using a two-sample $t$-test (where TARGET aberrations were identified in the matching FFPE sample and the quality of OncoScan data was sufficient). Samples were considered informative for the calculation of analytical sensitivities where TARGET aberrations were identified in matched FFPE samples and OncoScan data from the urinary DNA was of sufficient quality for accurate genomic analysis.

\section{Data availability}

All microarray data (both raw CEL files and processed OSCHP files) have been publicly submitted to the ArrayExpress database (https://www.ebi.ac.uk/ arrayexpress) under accession number E-MTAB-3841. ${ }^{23}$ Alongside the 
Table 1 Tumour staging data for 23 patients with UBC together with information on TARGET aberrations identified

\begin{tabular}{|c|c|c|c|c|c|c|c|}
\hline $\begin{array}{l}\text { Patient } \\
\text { number }\end{array}$ & $\begin{array}{l}\text { Tumour } \\
\text { stage }\end{array}$ & Grade & DNA source & $\begin{array}{l}\text { Number of } \\
\text { TARGET } \\
\text { aberrations } \\
\text { identified }\end{array}$ & $\begin{array}{l}\text { Sensitivity for } \\
\text { TARGET } \\
\text { mutations } \\
\text { present in } \\
\text { FFPE (\%) }\end{array}$ & Details of TARGET aberrations & Comments \\
\hline 1 & pT2+ & G3 & $\begin{array}{l}\text { FFPE tumour sections } \\
\text { Urine supernatant } \\
\text { Urine cell pellet }\end{array}$ & $\begin{array}{c}2 \\
2 \\
\text { NA }\end{array}$ & $\begin{array}{l}\text { NA } \\
100 \\
\text { NA }\end{array}$ & $\begin{array}{l}\text { KIT amp; CDKN2A/B biallelic loss } \\
\text { KIT amp; CDKN2A/B biallelic loss } \\
\text { NA }\end{array}$ & DNA insufficient for OncoScan \\
\hline 2 & pT2+ & G3 & $\begin{array}{l}\text { FFPE tumour sections } \\
\text { Urine supernatant } \\
\text { Urine cell pellet }\end{array}$ & $\begin{array}{l}5 \\
5 \\
0\end{array}$ & $\begin{array}{c}\text { NA } \\
100 \\
0\end{array}$ & $\begin{array}{l}\text { AKT2 amp; CDK4 amp; MCL1 amp; MDM2 } \\
\text { amp; RAF1 amp } \\
\text { AKT2 amp; CDK4 amp; MCL1 amp; MDM2 } \\
\text { amp; RAF1 amp } \\
\text { NA }\end{array}$ & \\
\hline 3 & pT2+ & G3 & $\begin{array}{l}\text { Urine supernatant } \\
\text { Urine cell pellet }\end{array}$ & 3 & $\begin{array}{l}67 \\
67\end{array}$ & $\begin{array}{l}\text { PIK3CA:c.1633G >A (p.(E545K)); PIK3CA: } \\
\text { c.1624G >A (p.(E542K)); } \\
\text { CDKN2A/B biallelic loss } \\
\text { PIK3CA:c.1633G >A (p.(E545K)); TP53: } \\
\text { c.524G >A (p.(R175H)); } \\
\text { CDKN2A/B biallelic loss } \\
\text { PIK3CA:C.1633G >A (p.(E545K)); } \\
\text { TP53:p.R175H; TP53:c.524G > } \\
\text { A (p.(R175H)) CDKN2A/B biallelic loss }\end{array}$ & $\begin{array}{l}\text { Differences in somatic mutations identified } \\
\text { in urine and FFPE samples highlights } \\
\text { tumour heterogeneity. Mutations in urine } \\
\text { cfDNA consistent with FFPE sections from } \\
\text { deeper tumour material (data not shown) }\end{array}$ \\
\hline 4 & pT2+ & G3 & $\begin{array}{l}\text { FFPE tumour sections } \\
\text { Urine supernatant } \\
\text { Urine cell pellet }\end{array}$ & $\begin{array}{l}0 \\
0 \\
0\end{array}$ & $\begin{array}{l}\text { NA } \\
\text { NA } \\
\text { NA }\end{array}$ & & $\begin{array}{l}\text { Clear and consistent CN aberrations evident } \\
\text { for both cfDNA and FFPE DNA }\end{array}$ \\
\hline 5 & pT2+ & G3 & $\begin{array}{l}\text { Urine supernatant } \\
\text { Urine cell pellet }\end{array}$ & 12 & 100 & $\begin{array}{l}\text { AKT2 amp; AURKA amp; BRAF amp; } \\
\text { CCND3 amp; CCNE1 amp; } \\
\text { CDK6 amp; CRKL amp; EGFR amp; FGFR1 } \\
\text { amp; MAPK1 amp; } \\
\text { MCL1 amp; MET amp } \\
\text { AKT2 amp; AURKA amp; BRAF amp; } \\
\text { CCND3 amp; CCNE1 amp; CDK6 amp; } \\
\text { CRKL amp; EGFR amp; FGFR1 amp; } \\
\text { MAPK1 amp; MCL1 amp; MET amp }\end{array}$ & $\begin{array}{l}\text { Apparently representative of germline gen- } \\
\text { ome only }\end{array}$ \\
\hline 6 & pT2+ & G3 & $\begin{array}{l}\text { FFPE tumour sections } \\
\text { Urine supernatant } \\
\text { Urine cell pellet }\end{array}$ & $\begin{array}{c}\text { NA } \\
1 \\
0\end{array}$ & $\begin{array}{l}\text { NA } \\
100 \\
0\end{array}$ & MCL1 amp & $\begin{array}{l}\text { DNA insufficient for OncoScan } \\
\text { In absence of FFPE results, assume cfDNA } \\
\text { to be representative of tumour } \\
\text { Apparently representative of germline gen- } \\
\text { ome only }\end{array}$ \\
\hline 7 & pT2+ & G3 & $\begin{array}{l}\text { FFPE tumour sections } \\
\text { Urine supernatant }\end{array}$ & $\begin{array}{l}0 \\
2\end{array}$ & $\begin{array}{l}\text { NA } \\
100 \\
100\end{array}$ & $\begin{array}{l}\text { Quality insufficient to call aberrations } \\
\text { CCND1 amp; CCNE1 amp } \\
\text { CCND1 amp; CCNE1 amp }\end{array}$ & $\begin{array}{l}\text { In absence of FFPE results, assume urine } \\
\text { DNA aberrations to be representative of } \\
\text { tumour } \\
\text { In absence of FFPE results, assume urine } \\
\text { DNA aberrations to be representative of } \\
\text { tumour }\end{array}$ \\
\hline 8 & pT2+ & G3 & $\begin{array}{l}\text { FFPE tumour sections } \\
\text { Urine supernatant } \\
\text { Urine cell pellet }\end{array}$ & $\begin{array}{l}3 \\
3 \\
1\end{array}$ & $\begin{array}{l}\text { NA } \\
100 \\
33\end{array}$ & $\begin{array}{l}\text { FGFR1 amp; MYC amp; PIK3CA: } \\
\text { c.3140A > G (p.(H1047R)) } \\
\text { FGFR1 amp; MYC amp; PIK3CA: } \\
\text { c.3140A > G (p.(H1047R)) } \\
\text { PIK3CA:c.3140A > G (p.(H1047R)) }\end{array}$ & \\
\hline 9 & pTa & G1 & $\begin{array}{l}\text { FFPE tumour sections } \\
\text { Urine supernatant } \\
\text { Urine cell pellet }\end{array}$ & $\begin{array}{l}0 \\
0 \\
0\end{array}$ & $\begin{array}{l}\text { NA } \\
\text { NA } \\
\text { NA }\end{array}$ & & \\
\hline 10 & pT2+ & G3 & $\begin{array}{l}\text { FFPE tumour sections } \\
\text { Urine supernatant } \\
\text { Urine cell pellet }\end{array}$ & $\begin{array}{c}0 \\
0 \\
N A\end{array}$ & $\begin{array}{l}\text { NA } \\
\text { NA } \\
\text { NA }\end{array}$ & & DNA insufficient for OncoScan \\
\hline 11 & pTa & G2 & $\begin{array}{l}\text { FFPE tumour sections } \\
\text { Urine supernatant } \\
\text { Urine cell pellet }\end{array}$ & $\begin{array}{l}1 \\
\text { NA } \\
\text { NA }\end{array}$ & $\begin{array}{l}\text { NA } \\
\text { NA } \\
\text { NA }\end{array}$ & CDKN2A biallelic loss & $\begin{array}{l}\text { Quality of OncoScan data too poor for } \\
\text { accurate analysis } \\
\text { DNA insufficient for OncoScan }\end{array}$ \\
\hline 12 & pT2+ & G3 & $\begin{array}{l}\text { FFPE tumour sections } \\
\text { Urine supernatant } \\
\text { Urine cell pellet }\end{array}$ & $\begin{array}{l}1 \\
1 \\
1\end{array}$ & $\begin{array}{l}\text { NA } \\
100 \\
100\end{array}$ & $\begin{array}{l}\text { TP53:c.844C > T (p.(R282W)) } \\
\text { TP53:c.844C > T (p.(R282W)) } \\
\text { TP53:c.844C>T (p.(R282W)) }\end{array}$ & \\
\hline 13 & pT2+ & $\mathrm{G} 1$ & $\begin{array}{l}\text { FFPE tumour sections } \\
\text { Urine supernatant } \\
\text { Urine cell pellet } \\
\text { FFPE tumour sections }\end{array}$ & $\begin{array}{l}3 \\
3 \\
3 \\
1\end{array}$ & $\begin{array}{l}\text { NA } \\
100 \\
100 \\
\text { NA }\end{array}$ & $\begin{array}{l}\text { CCND1 amp; CDK4 amp; MDM2 amp } \\
\text { CCND1 amp; CDK4 amp; MDM2 amp } \\
\text { CCND1 amp; CDK4 amp; MDM2 amp } \\
\text { PIK3CA:c.3140A > G (p.(H1047R)) }\end{array}$ & \\
\hline
\end{tabular}




\begin{tabular}{|c|c|c|c|c|c|c|c|}
\hline \multirow{5}{*}{$\begin{array}{l}\text { Patient } \\
\text { number }\end{array}$} & \multirow{5}{*}{$\begin{array}{c}\text { Tumour } \\
\text { stage }\end{array}$} & \multirow[b]{5}{*}{ Grade } & \multicolumn{4}{|c|}{ Sensitivity for } & \multirow[b]{5}{*}{ Comments } \\
\hline & & & & \multirow{4}{*}{$\begin{array}{c}\text { Number of } \\
\text { TARGET } \\
\text { aberrations } \\
\text { identified }\end{array}$} & TARGET & \multirow[b]{4}{*}{ Details of TARGET aberrations } & \\
\hline & & & & & mutations & & \\
\hline & & & & & present in & & \\
\hline & & & DNA source & & FFPE (\%) & & \\
\hline & & & Urine supernatant & 1 & 100 & PIK3CA:c.3140A > G (p.(H1047R)) & \\
\hline & & & Urine cell pellet & 1 & 100 & PIK3CA:c.3140A > G (p.(H1047R)) & \\
\hline 15 & pT2+ & G3 & FFPE tumour sections & 1 & NA & EGFR amp & \\
\hline & & & Urine supernatant & 0 & 0 & & \\
\hline & & & Urine cell pellet & 0 & & & \\
\hline \multirow[t]{5}{*}{16} & \multirow[t]{5}{*}{ pT1 } & \multirow[t]{5}{*}{ G3 } & FFPE tumour sections & 8 & NA & \multirow{5}{*}{\multicolumn{2}{|c|}{$\begin{array}{l}\text { BRAF amp; CCND3 amp; CDK6 amp; EGFR } \\
\text { amp; MAPK3 amp; MET amp; MYC amp; } \\
\text { RAF1 amp } \\
\text { BRAF amp; CCND3 amp; CDK6 amp; EGFR } \\
\text { amp; MAPK3 amp; MET amp; MYC amp; } \\
\text { RAF1 amp } \\
\text { CCND3 amp; MAPK3 amp; MYC amp; RAF1 } \\
\text { amp }\end{array}$}} \\
\hline & & & & & & & \\
\hline & & & Urine supernatant & 8 & 100 & & \\
\hline & & & & & & & \\
\hline & & & Urine cell pellet & 4 & 50 & & \\
\hline \multirow[t]{3}{*}{17} & \multirow[t]{3}{*}{ pT1 } & \multirow[t]{3}{*}{ G2 } & FFPE tumour sections & 1 & NA & \multirow{3}{*}{\multicolumn{2}{|c|}{$\begin{array}{l}\text { CCND1 amp } \\
\text { CCND1 amp } \\
\text { CCND1 amp }\end{array}$}} \\
\hline & & & Urine supernatant & 1 & 100 & & \\
\hline & & & Urine cell pellet & 1 & 100 & & \\
\hline \multirow[t]{5}{*}{18} & \multirow[t]{5}{*}{ pT2+ } & \multirow[t]{5}{*}{ G3 } & FFPE tumour sections & 4 & NA & \multirow{5}{*}{\multicolumn{2}{|c|}{$\begin{array}{l}\text { CCNE1 amp; RAF1 amp; CDKN2A/B bialle- } \\
\text { lic loss; PIK3CA:c.1624G > A } \\
\text { (p.(E542K)) } \\
\text { CCNE1 amp; RAF1 amp; CDKN2A/B bialle- } \\
\text { lic loss; PIK3CA:c.1624G > A } \\
\text { (p.(E542K)) } \\
\text { CCNE1 amp; RAF1 amp; CDKN2A/B bialle- } \\
\text { lic loss; PIK3CA:c.1624G > A } \\
\text { (p.(E542K)) }\end{array}$}} \\
\hline & & & Urine supernatant & 4 & 100 & & \\
\hline & & & & & & & \\
\hline & & & Urine cell pellet & 4 & 100 & & \\
\hline & & & & & & & \\
\hline \multirow[t]{3}{*}{19} & \multirow[t]{3}{*}{ pT2+ } & \multirow[t]{3}{*}{$\mathrm{G} 2$} & FFPE tumour sections & 3 & NA & \multirow{3}{*}{$\begin{array}{l}\text { CCND1 amp; CDKN2A/B biallelic loss; TSC1 } \\
\text { biallelic loss } \\
\text { CCND1 amp; CDKN2A/B biallelic loss } \\
\text { CCND1 amp; CDKN2A/B biallelic loss }\end{array}$} & \\
\hline & & & Urine supernatant & 2 & 67 & & \\
\hline & & & Urine cell pellet & 2 & 67 & & \\
\hline \multirow[t]{2}{*}{20} & \multirow[t]{2}{*}{$\mathrm{pTa}$} & \multirow[t]{2}{*}{ G3 } & FFPE tumour sections & 0 & NA & & \\
\hline & & & Urine supernatant & 0 & NA & & DNA insufficient for 0 coscan \\
\hline \multirow{3}{*}{21} & Ta & $c_{1}$ & & & & & \\
\hline & pla & GI & $\begin{array}{l}\text { FFPE tumour sections } \\
\text { Urine supernatant }\end{array}$ & 0 & $\begin{array}{l}\text { NA } \\
\text { NA }\end{array}$ & & \\
\hline & & & Urine cell pellet & 0 & NA & & \\
\hline 22 & pTa & G3 & FFPE tumour sections & 1 & NA & CDKN2A/B biallelic loss & \\
\hline & & & Urine supernatant & 1 & 100 & CDKN2A/B biallelic loss & DNA incufficint for $\mathrm{OnceScap}$ \\
\hline & & & & & & & \\
\hline 23 & pT1 & G2 & FFPE tumour sections & 1 & NA & CDKN2A/B biallelic loss & \\
\hline & & & $\begin{array}{l}\text { Urine supernatant } \\
\text { Urine cell pellet }\end{array}$ & $\begin{array}{l}1 \\
1\end{array}$ & 100 & CDKN2A/B biallelic loss & \\
\hline
\end{tabular}

Abbreviations: FFPE, formalin-fixed paraffin embedded; NA, not applicable.

Analytical sensitivity (for detection of FFPE-identified aberrations) is indicated. CN probes are mapped to Genome Reference Consortium human genome build 37 (GRCh37). Reference sequences used for somatic mutations listed are TP53 (NM_000546.5) and PIK3CA (NM_006218.2).

microarray data, pertinent meta-data has also been included detailing the tumour grading/staging and anonymised patient of sample origin.

\section{Orthogonal validation of somatic mutations}

Where DNA availability allowed, SM calls made by the OncoScan assay were verified by PCR and next-generation sequencing. Sequencing was performed on a MiSeq (Illumina, San Diego, CA, USA) and data analysed by a custom pipeline built from cutadapt version 1.2.1.24 BWA-MEM version 0.7.12, ${ }^{25}$ SAMtools version $1.2^{26}$ and VarScan version 2.3.9..$^{27}$

\section{RESULTS}

Genomic profiles, where informative, show clear agreement of $\mathrm{CN}$ profiles between corresponding patient samples (Table 1; Figure 1). Tumour heterogeneity is evidenced by identification of disparate SMs and $\mathrm{CN}$ aberrations in matched samples (Table 1; patient 3), as well as by inconsistent aberrant levels for matched abnormalities in corresponding patient samples.

Somatic mutations identified by the OncoScan assay were validated using next-generation sequencing approaches as described above. Depending on DNA availability, mutations were confirmed either in remaining urine and FFPE DNAs or using DNA extracted from fresh-frozen tissue taken from the same patient tumours. This analysis allowed for confirmation of all mutations that were identified in all matched FFPE and urine DNA samples assayed. Only the TP53 mutation in patient 3 (known to be clonal due to its absence in the initial FFPE DNA investigated) could not be identified in the matched fresh-frozen material and unfortunately there was insufficient FFPE or urine DNA available for this patient to allow further testing. However, 




Figure 1 Example of genomic profiles from DNAs extracted from FFPE and cellular and cfDNA from the urine from patient 23 with a stage G2 pT1 NMIBC. Comparison of profiles shows consistent aberrations identified in all three samples (red=loss, blue = gain and yellow $=\mathrm{LOH}$ ). Aberrations from all three sources show homozygous loss at 9p21.3 including CDKN2A/B; listed in the TARGET database. Biallelic inactivation of CDKN2A/B may predict response to CDK4/6 inhibitors for this patient.


Figure 2 By examining the BAF plots of the SNP probes for patient 13, the highest aberrant cell load for this patient is observed in cfDNA (greatest separation in bottom plots). The number of TARGET aberrations (3; amplifications of CCND1 (may predict sensitivity to CDK4/6 inhibitors), MDM2 (may predict sensitivity to Nutlins and MDM2 inhibitors) and CDK4 (may predict sensitivity to CDK4/6 inhibitors)) is consistent across all sample types. A degree of tumour heterogeneity is also clear.

as this mutation was identified independently in three separate DNAs from this patient using the OncoScan assay, we are confident that this is not an artefactual finding.

The tumour genome burden of the cfDNA and cellular DNA was calculated from the BAF of heterozygous SNPs and the predicted tumour genome burdens of urinary DNA samples were compared using a paired $t$-test (Materials and Methods). This data demonstrate a significantly greater tumour genome burden and lower germline DNA contamination in cfDNA over cellular DNA $(P<0.001)$. These differences were also visualised in BAF plots, showing a lower detection rate of targetable genomic biomarkers in urinary cellular DNA. Furthermore, in six samples, cellular DNA was insufficient for elucidation of appropriate genomic information using the OncoScan FFPE assay kit (Table 1); in contrast, 22/23 urine supernatants studied provided sufficient cfDNA for accurate characterisation of genomic aberrations.

In several samples, cfDNA from urine supernatant was observed as having a greater aberrant tumour genome load than FFPE material (Figures 2 and 3). For patient 6 (Table 1), there was insufficient DNA from FFPE tumour material to yield a result; only the cfDNA sample from this patient evidenced the cancer genome.

Analytical sensitivities for detection of key FFPE-identified genomic biomarkers were calculated, as described above. These data show cfDNA to have an average analytical sensitivity of $\sim 90 \%$ (range $0-100 \%$, 17 informative samples) for detection of FFPE-identified aberrations. Cellular DNA from the urine has an average analytical sensitivity of $61 \%$ (range 0-100\%, 15 informative samples). These data demonstrate a significantly greater analytical sensitivity for cfDNA over cellular DNA from urine $(P<0.04$; Table 1$)$.

Both the FFPE samples and cfDNA from urine, which were examined in our data set, showed an average of 2.3 TARGET aberrations per patient (range 0-12, 22 samples informative). DNA from urine cell pellets showed an average of 1.3 TARGET aberrations per patient (range 0-4, 18 samples informative; Table 1).

Following blinded analysis, 11 out of 12 urinary cfDNAs from nonUBC patients showed genomics consistent with the germline genome only. One patient, later revealed to harbour prostatic duct carcinoma, showed CN alterations consistent with malignancy (Figure 4). 

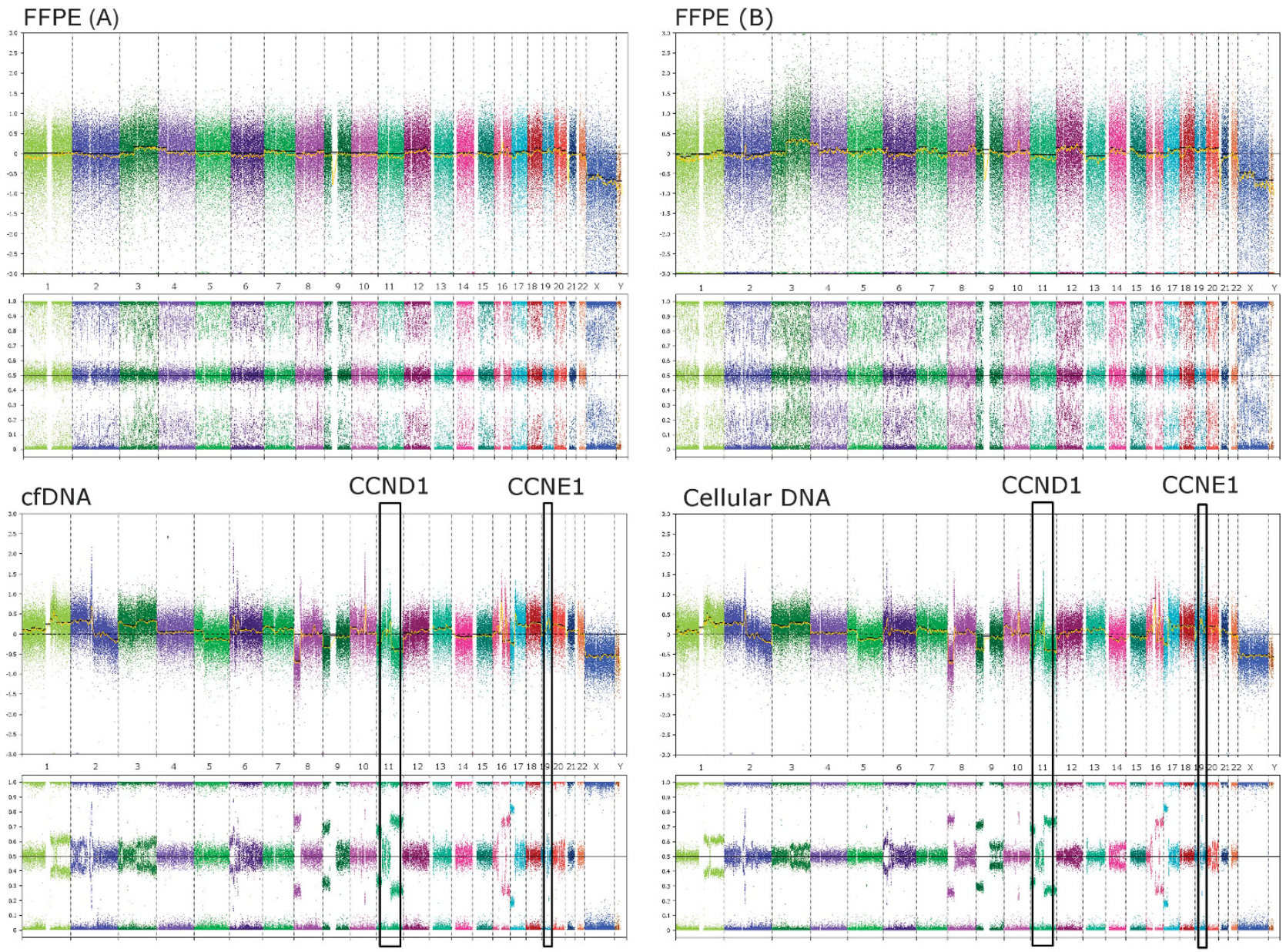

Figure 3 Patient 7. DNAs extracted from urine provide improved quality genomic data and clearer characterisation of the tumour profile than DNA from FFPE tumour material, despite repeat slides being cut and extracted. Two TARGET aberrations (CCND1 amplification (may predict sensitivity to CDK4/6 inhibitors) and CCNE1 amplification (may predict sensitivity to CDK2 inhibitors)) were observed in both cfDNA and urinary cellular DNA however none of these aberrations were independently called in two separate DNAs from FFPE tumour material.

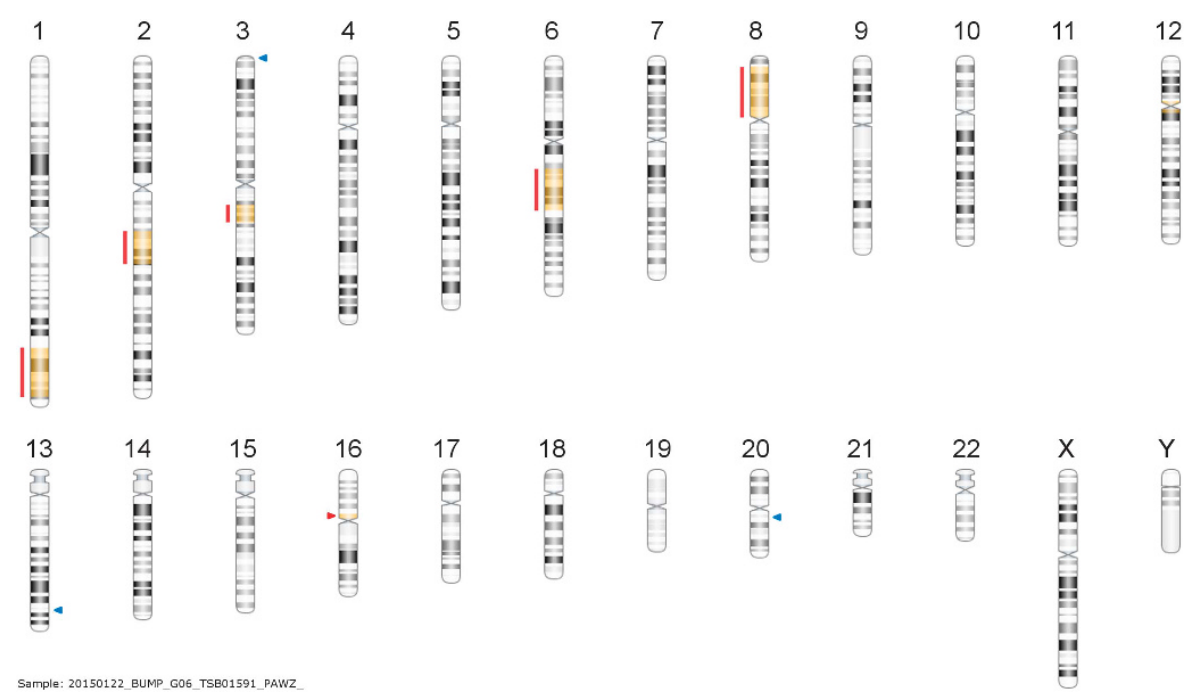

Figure 4 Genomic profile from urinary cfDNA of non-UBC patient with prostatic duct carcinoma confirms that detection of a tumour genome in urinary DNA is not bladder cancer specific. Red lines highlight regions of CN loss with resulting loss of heterozygosity indicated by yellow colouring of the chromosomal regions. Blue and red arrows indicate sub-microscopic germline $\mathrm{CN}$ gains and losses, respectively. 


\section{DISCUSSION}

Recent developments in the genomic profiling of tumours have led to rapid growth in our understanding of the genetic basis of cancer. ${ }^{28}$ Genomic biomarkers are increasingly being identified as indicators for disease prognosis or diagnosis, or for predicting response to targeted therapies. ${ }^{22}$ Non-invasive identification of these biomarkers is an area of intense interest and shows potential to significantly improve patient care. Many groups have looked at cell-free tumour DNA circulating in the blood or other body fluids as important liquid biopsy resources for non-invasive tumour profiling ${ }^{9,15}$ and, recurrently, specific genomic biomarkers for UBC diagnosis have been identified in cells exfoliated into the urine.

Here we demonstrate the clinical utility of urinary DNA from UBC patients and support the hypothesis that cfDNA from urine provides an improved resource for non-invasive genomic profiling of UBC when compared with urine cellular DNA. A previous study looking more simply at microsatellite analyses in urinary DNAs from 44 UBC patients suggested a higher analytical sensitivity when using cfDNA over DNA from urinary cell pellets. ${ }^{15}$ Here we have elaborated on these findings and illustrated the use of cfDNA to capture genomic complexity across the UBC tumour genome using a comprehensive genomic profiling platform well suited to small quantities of highly degraded DNA. Our data show that cfDNA from UBC patients is highly representative of the tumour genome and has a consistently higher tumour burden than DNA from exfoliated whole cells $(P<0.001)$, allowing greater detection of important tumourspecific biomarkers $(P<0.04)$. It is hypothesised that this increased representation of the tumour genome in the cfDNA is a result of the increased rate of necrosis for tumour cells relative to normal urothelium.

Our data show evidence of tumour heterogeneity, illustrated by variations in genomics between matched patient samples (all taken at diagnosis). Our data also demonstrate that biomarkers present in FFPE material can be accurately and robustly identified in urinary cfDNA with a higher analytical sensitivity (90\%) than urine cellular DNA (61\%), $P<0.04$.

Analyses of cfDNAs from control patients with UBC symptoms show CN aberrations only in the presence of other malignancies. These data suggest high specificity of urinary cfDNA for the detection of malignancy, but confirms that genomic analysis of cfDNA is not UBC specific. Previous authors have investigated nucleic-acid-based biomarkers in the body fluids of patients with a variety of urologic malignancies, recently reviewed by Ralla et al. ${ }^{9}$

Genomic CN aberrations in exfoliated cells from urine have recently been shown to pre-date cancer development by $\sim 3$ years in some patients; ${ }^{29}$ it is possible that cfDNA would not mirror these predictive $\mathrm{CN}$ aberrations in the absence of necrotic tumour material. The investigation of such phenomena was beyond the remit of this study, but is the subject of ongoing analyses.

The data presented herein represent a proof of principle that urinary cfDNA from UBC patients represents a promising resource for the identification of complex cancer genomes and specific targetable aberrations when profiled using the OncoScan FFPE assay kit. Urinary cfDNA may thus have utility for non-invasive disease diagnosis, surveillance, prognostication and prediction or monitoring of treatment responses.

\section{CONCLUSIONS}

cfDNA from urine supernatant allows for accurate and detailed wholegenome profiling of UBCs. It has a higher tumour genome burden than urine cellular DNA, and shows a higher analytical sensitivity for detection of important genomic biomarkers present in the tumour genome. $\mathrm{cfDNA}$ from the urine thus provides a promising resource for non-invasive genomic profiling of UBCs to help guide patient management without invasive sampling, thereby potentially improving patient care.

\section{CONFLICT OF INTEREST}

RTB has previously contributed to an advisory board for Olympus Medical Systems with regard to narrow band imaging cystoscopy. JMF, AO and KGS are all paid employees of Affymetrix UK Ltd and hold shares in Affymetrix (NASDAQ: AFFX). The remaining authors declare no conflict of interest.

\section{ACKNOWLEDGEMENTS}

BCPP is funded by Cancer Research UK, the University of Birmingham and the Birmingham \& The Black Country and West Midlands North and South Comprehensive Local Research Networks, and sponsored by the University of Birmingham. The BCPP biospecimen collection is supported by funding from the Birmingham Experimental Cancer Medicine Centre. DGW is funded by a philanthropic donation to the University of Birmingham in support of bladder cancer research. We acknowledge the West Midlands Regional Genetics Laboratory, the Technology Strategy Board (now Innovate-UK) Stratified Medicine Innovation Platform for funding this research via grant number 101032, and Affymetrix UK Limited. We also like to acknowledge the work of Henry Wood, Morag Taylor and Professor Philip Quirke from Leeds Institute of Cancer and Pathology for the design and undertaking of targeted NGS of FFPE and urine DNA samples to confirm the presence of OncoScan-identified somatic mutations. They also wish to extend their gratitude to Elizabeth Bradshaw for her help in formatting of this article.

1 Ploeg M, Aben KKH, Kiemeney LA: The present and future burden of urinary bladder cancer in the world. World J Urol 2009; 27: 289-293.

2 Schrier BP, Hollander MP, van Rhijn BWG, Kiemeney LALM, Witjes JA: Prognosis of muscle-invasive bladder cancer: difference between primary and progressive tumours and implications for therapy. Eur Urol 2004; 45: 292-296.

3 Moschini M, Sharma V, Dell'oglio P, Cucchiara V, Gandaglia G, Cantiello F et al: Comparing long-term outcomes between primary versus progressive muscle invasive bladder cancer after radical cystectomy. BJU Int 2015. e-pub ahead of print 7 April 2015; doi:10.1111/bju.13146.

4 Witjes JA, Compérat E, Cowan NC, De Santis M, Gakis G, Lebret T et al: EAU guidelines on muscle-invasive and metastatic bladder cancer: summary of the 2013 guidelines. Eur Urol 2014; 65: 778-792.

5 Knowles MA, Hurst CD: Molecular biology of bladder cancer: new insights into pathogenesis and clinical diversity. Nat Rev Cancer 2014; 15: 25-41.

6 The Cancer Genome Atlas Research Network: Comprehensive molecular characterization of urothelial bladder carcinoma. Nature 2014; 507: 315-322.

7 Chekaluk Y, Wu C-L, Rosenberg J, Riester M, Dai Q, Lin S et al: Identification of nine genomic regions of amplification in urothelial carcinoma, correlation with stage, and potential prognostic and therapeutic value. PloS One 2013; 8: e60927.

8 Hurst CD, Platt FM, Taylor CF, Knowles MA: Novel tumor subgroups of urothelial carcinoma of the bladder defined by integrated genomic analysis. Clin Cancer Res 2012; 18: 5865-5877.

9 Ralla B, Stephan C, Meller S, Dietrich D, Kristiansen G, Jung K: Nucleic acid-based biomarkers in body fluids of patients with urologic malignancies. Crit Rev Clin Lab Sci 2014; 51: 200-231.

10 Bryan RT, Kirby R, Mostafid H: Does the nonurologic scientific community understand urothelial bladder cancer? Eur Urol 2014; 66: 601-602.

11 Têtu B: Diagnosis of urothelial carcinoma from urine. Mod Pathol 2009; 22 (Suppl 2): S53-S59.

12 Halling KC, Kipp BR: Bladder cancer detection using FISH (UroVysion assay). Adv Anat Pathol 2008; 15: 279-286.

13 Cussenot O, Sighar K, Mohammed M, Hugonin S, Ondet V, Larre S et al: Detection of specific chromosomal aberrations in urine using BCA-1 (oligo-CGH-array) enhances diagnostic sensitivity and predicts the aggressiveness of non-muscle-invasive bladder transitional cell carcinoma. World J Urol 2014; 32: 551-557.

14 Larré S, Camparo P, Comperat E, Gil Diez De Medina S, Traxer O, Roupret M et al: Diagnostic, staging, and grading of urothelial carcinomas from urine: performance of BCA-1, a mini-array comparative genomic hybridisation-based test. Eur Urol 2011; 59: 250-257.

15 Szarvas T, Kovalszky I, Bedi K, Szendroi A, Majoros A, Riesz P et al: Deletion analysis of tumor and urinary DNA to detect bladder cancer: urine supernatant versus urine sediment. Oncol Rep 2007; 18: 405-409. 
16 Stroun M, Maurice P, Vasioukhin V, Lyautey J, Lederrey C, Lefort F et al: The origin and mechanism of circulating DNA. Ann NY Acad Sci 2000; 906: 161-168.

17 Pantel K, Alix-Panabières C: Real-time liquid biopsy in cancer patients: fact or fiction? Cancer Res 2013; 73: 6384-6388.

18 Chang HW, Tsui KH, Shen LC, Huang HW, Wang SN, Chang PL: Urinary cell-free DNA as a potential tumor marker for bladder cancer. Int J Biol Markers 2007: 22: 287-294.

19 Zeegers MP, Bryan RT, Langford C, Billingham L, Murray P, Deshmukh NS et al: The West Midlands Bladder Cancer Prognosis Programme: rationale and design. BJU Int 2010; 105: 784-788.

20 Hardenbol P, Banér J, Jain M, Nilsson M, Namsaraev EA, Karlin-Neumann GA et al: Multiplexed genotyping with sequence-tagged molecular inversion probes. Nat Biotechnol 2003; 21: 673-678.

21 Foster JM, Oumie A, Togneri FS, Vasques FR, Hau D, Taylor M et al: Cross-laboratory validation of the OncoScan ${ }^{\circledR}$ FFPE Assay, a multiplex tool for whole genome tumour profiling. BMC Med Genomics 2015; 8: 5.

22 Van Allen EM, Wagle N, Stojanov P, Perrin DL, Cibulskis K, Marlow S et al: Wholeexome sequencing and clinical interpretation of formalin-fixed, paraffin-embedded tumor samples to guide precision cancer medicine. Nat Med 2014; 20: 682-688.

23 Kolesnikov N, Hastings E, Keays M, Melnichuk O, Tang YA, Williams E et al: ArrayExpress update - simplifying data submissions. Nucleic Acids Res 2015; 43: D1113-D1116.

24 Martin M: Cutadapt removes adapter sequences from high-throughput sequencing reads. EMBnet.journal 2011; 17: 10-12.

$25 \mathrm{Li} \mathrm{H}$ : Aligning sequence reads, clone sequences and assembly contigs with BWA-MEM. arXiv 2013; 1303: 3997.
26 Li H, Handsaker B, Wysoker A, Fennell T, Ruan J, Homer N et al: The Sequence Alignment/Map format and SAMtools. Bioinformatics 2009; 25: 2078-2079.

27 Koboldt DC, Zhang Q, Larson DE, Shen D, McLellan MD, Lin L et al: VarScan 2: somatic mutation and copy number alteration discovery in cancer by exome sequencing. Genome Res 2012; 22: 568-576.

28 Ciriello G, Miller ML, Aksoy BA, Senbabaoglu Y, Schultz N, Sander C: Emerging landscape of oncogenic signatures across human cancers. Nat Genet 2013; 45: 1127-1133.

29 Bonberg N, Pesch B, Behrens T, Johnen G, Taeger D, Gawrych K et al: Chromosomal alterations in exfoliated urothelial cells from bladder cancer cases and healthy men: a prospective screening study. BMC Cancer 2014; 14: 854

This work is licensed under a Creative Commons Attribution 4.0 International License. The images or other third party material in this article are included in the article's Creative Commons license, unless indicated otherwise in the credit line; if the material is not included under the Creative Commons license, users will need to obtain permission from the license holder to reproduce the material. To view a copy of this license, visit http:// creativecommons.org/licenses/by/4.0/ 